\title{
Colposcopy at the University Hospital in Conakry: Role of Colposcopy in Screening and/or Diagnosis of Cervical Cancer in the UTH University Teaching Hospital of Conakry
}

\author{
A. F. M. Soumah ${ }^{*}$, O. H. Bah ${ }^{2}$, E. M. Bah ${ }^{2}$, M. K. Camara², N. Keita ${ }^{2}$, A. Diallo ${ }^{3}$ \\ ${ }^{1}$ Gynecology Obstetrics Department, Teaching Hospital of Ignace Deen, Conakry, Guinea \\ ${ }^{2}$ Gynecology Obstetrics Department, Teaching Hospital of Donka, Conakry, Guinea \\ ${ }^{3}$ Jhpiego, Guinea \\ Email: *aboubacarfodemomo@gmail.com, oumouhawa.bah@jhpiegi.org, elhadjlalya70@yahoo.fr, \\ camarakantara@gmail.com,namoryk2010@yahoo.fr, abdoulaye.diallo@jhpiego.org
}

How to cite this paper: Soumah, A.F.M., Bah, O.H., Bah, E.M., Camara, M.K., Keita, N. and Diallo, A. (2018) Colposcopy at the University Hospital in Conakry: Role of Colposcopy in Screening and/or Diagnosis of Cervical Cancer in the UTH University Teaching Hospital of Conakry. Open Journal of Obstetrics and Gynecology, 8, 497-504. https://doi.org/10.4236/ojog.2018.85056

Received: January 24, 2018

Accepted: May 11, 2018

Published: May 14, 2018

Copyright (c) 2018 by authors and Scientific Research Publishing Inc. This work is licensed under the Creative Commons Attribution International License (CC BY 4.0).

http://creativecommons.org/licenses/by/4.0/

\begin{abstract}
Objective: This study was conducted to evaluate the role of colposcopy in the screening of cervical cancer at the UTH in Conakry. Material and Methods: We conducted a descriptive study on over a period of 18 months of from the 1 July 2004 to December 31, 2005 in the CHU of Conakry Any women aged from 25 to 65 years old who has agreed to the screening of cervical cancer by colposcopy was included. Statistical tests of sensitivity, specificity, and predictive values positive and negative were computed directly and compare among all participants. Results: During the study period, we included a total of 9339 women. For all precancerous and cancerous lesions, sensitivity and specificity were $93 \%$ and $64 \%$ respectively. With a positive predictive value of $46 \%$ and the negative predictive value of $90 \%$, low and high grade precancerous lesions were found with: a sensitivity of $91 \%$, the specificity of $64 \%$. The positive predictive value of $37 \%$, and the negative predictive value of $91 \%$ were estimed. Intra epithelial lesions (LIEBG) low-grade squamous sensitivity was $94 \%$, specificity of $62 \%$, the positive predictive value of $31 \%$, the negative predictive value of $95 \%$. For lesions intra epithelial highgrade squamous (LIEHG), the sensitivity was $85 \%, 10 \%$, the positive predictive value of $74 \%$ specificity, negative predictive value of $95 \%$. Conclusion: Colposcopy is a reference method of detection of precancerous lesions of the cervix to promote cancer.
\end{abstract}

\section{Keywords}

Cervical Cancer, Screening, Colposcopy 


\section{Introduction}

Cancer of the cervix is the second cancer of women in the world after the breast [1]. In developed countries, the incidence and mortality of cancer decreased considerably through systematic screening programs [2]. In Africa, on the other hand, cervical cancer mortality is about 22.5 deaths per 100,000 women per year and survival at 5 years is estimated at $21 \%$ [3]. In Guinea, cancer of the cervix is a real public health problem; it represents the first cancer of women, and the second all sex confused according to EDS Guinea 2012 [4]. Colposcopy is the screening tool commonly used in developing countries, which will also allow biopsy for the diagnosis. The objective of this work was to evaluate the role of colposcopy in screening for cancer of the cervix in the CHU of Conakry.

\section{Material and Methods}

In this study, written consents were obtained from all adult women participants including a research approval from the Gynecology and obstetrics chair of the University of Conakry. It is a cross-sectional, descriptive study for 18 months from 1St July 2004 to 31 December 2005 in the Gynecology-obstetrics services and laboratory of pathological anatomy of the CHU of Conakry. The study population consisted of women aged between 25 and 65 who has agreed to screening for cancer of the cervix by colposcopy. Detection was done by trained colposcopists and trained through a colposcopic examination with the application of acetic acid and the lugol. Biopsies were then made and sent to the laboratory of pathology in patients with 03 points or more in the depreciation of Reid [4].

\subsection{Inclusion Criterion}

Females aged 25 to 65 apparently healthy having visual screening test (positive or negative) after Signing informed consent.

\subsection{Criterion of Non-Inclusion}

Women under 25 and over 65 and all other women who have had a total hysterectomy.

Immediate treatment was proposed based on the extended and lesion situation compared to the cervical canal. Epi-info software was used for data analysis, statistical tests of sensitivity, specificity, predictive values, positive and negative were computed directly and compared among all participants.

\subsection{Limitations}

Colposcopy is not well indicated for screening, the use of Visual Acid Screening seems the appropriate technique for mass screening.

The absence of radiotherapy is an obstacle for the management of advanced cancers.

\section{Results}

1) Sociodemographic characteristics of the study population 
In total we have recruited 9339 women of which $89.6 \%$ were of reproductive age. In sociodemographic terms, the majority age group was that of $30-39$ years with an average of 45 . Soit une frequence de $48 \%$ in addition, $44.2 \%$ of women were illiterate contre $55.8 \%$ de Lettered woman and $49.9 \%$ were large multiparous.

\section{2) Exploration}

Visibility of the squamo-cylindrical junction (Table 1): our study had shown a lack of visibility of the area of junction in the $50-59$ age group and 60 65 years of $52.7 \%$ and $74.3 \%$ respectively (Table 2). Comparison of the results of colposcopy and histopathology: colposcopy was normal in $88.9 \%$ compared to histology which was normal in $63.2 \%$ (Table 3).

Concordance between the results of the colposcopy and histology: for164 results of recognized colposcopies normal histology had found 7 LIEBG, 9 LIEHG and 3 cancers. The detection of the LIEBG rate was 42 per 1000 women. The detection of the LIEHG rate was 9 per 1000 women (Table 4).

Performance of colposcopy in the diagnosis of LIEBG, LIEHG and cancer: on all injuries precancerous and cancerous, the sensitivity was $93 \%$; specificity of $64 \%$, the positive predictive value of $46 \%$ and the negative predictive value of $90 \%$ (Table 5).

Table 1. Sociodemographic characteristics of the study population.

\begin{tabular}{|c|c|c|}
\hline Female age (years) & Workforce $(n=9339)$ & Percentage (\%) \\
\hline Age $25-29$ & 991 & 10.6 \\
\hline $30-39$ & 4492 & 48.1 \\
\hline $40-49$ & 2908 & 31.1 \\
\hline $50-59$ & 868 & 9.3 \\
\hline $60-65$ & 80 & 0.9 \\
\hline \multicolumn{3}{|l|}{ Gestity } \\
\hline 0 & 621 & 6.7 \\
\hline $1-2$ & 1837 & 19.7 \\
\hline $3-4$ & 2219 & 23.8 \\
\hline $5+$ & 4662 & 49.9 \\
\hline Women of childbearing age & 8363 & 89.54 \\
\hline \multicolumn{3}{|l|}{ Marital status } \\
\hline Bride & 6858 & 73.4 \\
\hline Widow & 367 & 4 \\
\hline Divorcee & 237 & 2.5 \\
\hline Other+ & 196 & 2.1 \\
\hline Unknown & 1681 & 18 \\
\hline Lettered woman & 5209 & 55.8 \\
\hline Woman illphabetes & 4126 & 44.2 \\
\hline
\end{tabular}


Table 2. Visibility of the cylindrical squamo junction over the age.

\begin{tabular}{cccccc}
\hline Groupe Age & Visible & pourcentage & Non visible & Pourcentage & Total \\
\hline $25-29$ & 377 & 64.0 & 212 & 36.0 & 589 \\
$30-39$ & 3113 & 82.9 & 644 & 17.1 & 3757 \\
$40-49$ & 1690 & 67.3 & 822 & 32.7 & 2512 \\
$50-59$ & 345 & 47.3 & 385 & 52.7 & 730 \\
$60-65$ & 18 & 25.7 & 52 & 74.3 & 70 \\
Total & $\mathbf{5 5 4 3}$ & $\mathbf{7 2 . 4}$ & $\mathbf{2 1 1 5}$ & $\mathbf{2 7 . 6}$ & $\mathbf{7 6 5 8}$ \\
\hline
\end{tabular}

Table 3. Distribution of the results of colposcopy and histopathology.

\begin{tabular}{ccccc}
\hline & Colposcopy & \multicolumn{3}{c}{ Histology } \\
\hline & number & $\%$ & number & $\%$ \\
\hline Normal & 8263 & 88.9 & 632 & 63.2 \\
Inconclusive & 237 & 2.6 & 37 & 3.7 \\
Atypical & & & 15 & 1.5 \\
LIEBG+ & 678 & 7.3 & 146 & 14.6 \\
LIEHG++ & 62 & 0.7 & 79 & 7.9 \\
Cancer & 54 & 0.6 & 91 & 9.1 \\
\hline
\end{tabular}

LIEBG+: Injury intra epithelial squamous to low grade; LIEHG++: Injury intra epithelial squamous of high grade.

Table 4. Concordance between the results of the colposcopy and histopathology.

\begin{tabular}{ccccc}
\hline Colposcopy & \multicolumn{5}{c}{ Histology } \\
\hline Normal & LIEBG & LIEHG & Cancer & Total \\
\hline 164 & 7 & 9 & 3 & 183 \\
273 & 115 & 34 & 11 & 433 \\
9 & 4 & 19 & 20 & 62 \\
2 & 1 & 0 & 48 & 51 \\
\hline
\end{tabular}

Table 5. Performance of colposcopy in the diagnosis of LIEBG, L IEHG and cancers.

\begin{tabular}{cccc}
\hline Result & Diseanse presents & Absent desease & Total \\
\hline positive Colposcopy & 252 & 294 & 546 \\
négative Colposcopy & 19 & 164 & 183 \\
Total & 271 & 458 & \\
\hline
\end{tabular}

Sensitivity $=252 / 271=0.93$ or $93 \%$, specificity $=294 / 458=0.64$ or $64 \%$; Positive predictive value $=252 / 546$ $=0.46$, or $46 \%$, value predictive negative $=164 / 183=0.90$ or $90 \%$.

The colposcopy was positive in $96,34 \%$ of cancer cases and falsely negative in $3.65 \%$ cases. Where a sensitivity of $96 \%$ a specificity of $98 \%$ and the predictive positive value and negative 96 percent and 98 percent respectively of colposcopy performance (Table 6). 
Table 6. Performance of colposcopy in the diagnosis of the LIEBG.

\begin{tabular}{cccc}
\hline & Disease presents & Absent disease & Total \\
\hline Positive colposcopy & 120 & 273 & 393 \\
Negative colposcopy & 7 & 164 & 171 \\
& 127 & 437 & 564 \\
\hline
\end{tabular}

Sensitivity $=120 / 127=0.94$ or $94 \%$, specificity $=273 / 437=0.62$ or $62 \%$; Positive predictive value $=120 / 393$ $=0.31$ or $31 \%$, value predictive negative $=164 / 171=0.95$ or $95 \%$.

\section{3) Treatment}

Cryotherapy was carried out at $11.8 \%$ of women with the LIEBG and $49.4 \%$ of women with the LIEHG. The cone was made on 39\% cases of LIEHG and $2.1 \%$ of micro-invasive cancer. Surgery was also made on $8.5 \%$ of cancer (Figure 1 ).

\section{Discussions}

During this work, the objective was to assess the role of colposcopy in screening for cancer of the cervix in the CHU of Conakry. We were able to collect a total of 9339 patients at the waning of organized screening.

1) Sociodemographic characteristics (Table 1)

Women of childbearing age were more represented in the age group of 30 - 39 years at $48 \%$.

Our results were inferior to those reported by Ben Aissa et al. who were $68.4 \%$ in the $30-49$ age group of screened women [5]. In our series, this rate can be explained by the beginning of this organized free screening activity, associated with a large media campaign with low level of perception of the population. As for the gestity, large multigestes accounted for nearly half of our study population; this can be explained by the predisposition of the great multipara to injuries precancerous and cancer of the cervix [1]. Compared to the level of education, $55.8 \%$ of the women recruited were applied which could think that they had a better understanding of the importance and desirability of a champagne of organized screening. These patients were either married women $73.4 \%$ majority. This observation would be close to that of Tebeu and al in Cameroon who were 73.5\% [6]. Thus showing the impact and the place of the spouse on the health of the partner as we revealed several authors in the literature [7].

\section{2) Exploration}

Visibility of the cylindrical age squamo junction (Table 2). Our study had shown a lack of full visibility of the junction box in the age group $50-59$ years $60-65$ years of $47.3 \%$ and $25.7 \%$ respectively. It is established that over the age of the woman stopped there are difficult to fully see the squamo-cylindrical junction [6], our results joined those of Baker et al. indicating that the percentage of junction not seen past of $11.5 \%$ before $50 \%$ to $75 \%$ beyond [6].

\section{Colposcopy and histology (Table 3)}

Colposcopy and histology were normal $88.9 \%$ and $63.2 \%$, respectively in our series. Precancerous lesions have been highlighted in $8 \%$ of women. The diagnosis 


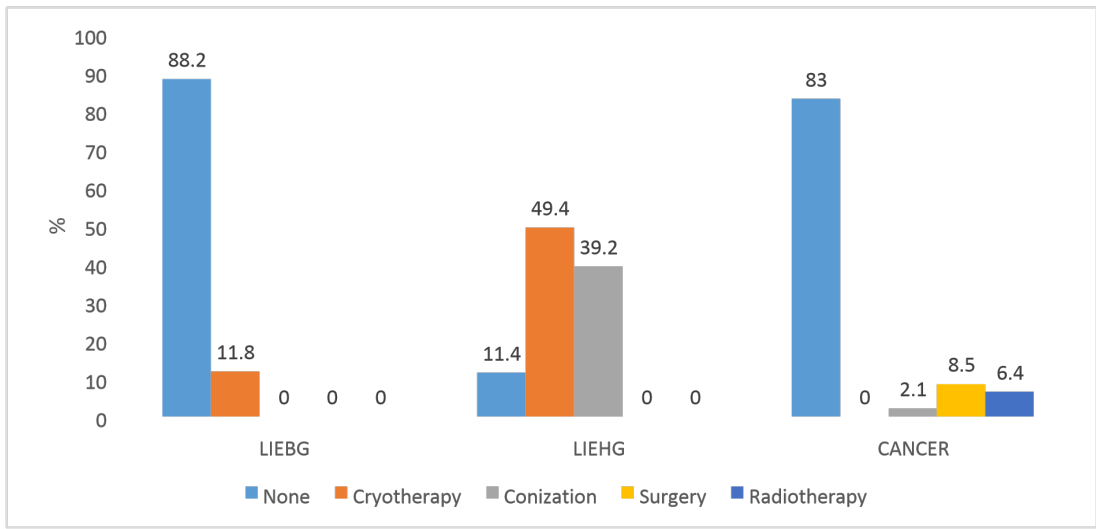

(a)

\begin{tabular}{|c|c|c|c|c|c|}
\hline & Cryotherapie & Conization & Surgery & Radiotherapie & None \\
\hline LIEBG & 11.8 & 0 & 0 & 0 & 88.2 \\
\hline LIEHG & 49.4 & 39.2 & 0 & 0 & 11.4 \\
\hline Cancer & 0 & 2.1 & 8.5 & 6.4 & 83 \\
\hline
\end{tabular}

(b)

Figure 1. Type of treatment provided.

of cancer was established at $0.6 \%$ is a detection rate of 6 in 1000. The LIEBG have been found in $14,6 \%$ of the cases examined, the LIEHG in $7.9 \%$ and invasive cancers in $9.1 \%$ of biopsies performed. Ritter et al. observed 8.9 percent of precancerous lesions and cancer $0.7 \%$ and $2 \%$ of colposcopy inconclusive [8].

With regard to the agreement between the results of colposcopy and histology (Table 4), for 164 recognized colposcopy results normal, histology had found 7 LIEBG, 9 LIEHG and 3 cancers. The detection of the LIEBG rate was 42 per 1000 women and 9 for 1000 women for the LIEHG. For all of the precancerous and cancerous lesions, sensitivity was $93 \%$; the specificity of $64 \%$, the positive predictive value of $46 \%$ and the negative predictive value of $90 \%$ (Table 5). Our study was stackable has money and al which showed a rise in the specificity and positive predictive value, what molecule as the SCC have tendencies to repeat tests for low grade lesions [9]. For only bottom and high-grade precancerous lesions (Table 6): the sensitivity was $94 \%$, specificity is $62 \%$, the positive predictive value of $31 \%$, the negative predictive value of $95 \%$. These results show a superiority of the colposcopy with biopsy in the detection of the LIEHG. However, these results should be viewed with caution, since it is well established that the reference of the precancerous and cancerous lesions diagnostic test remains [10] histology. In this study it was trained colposcopists, the results should not be generalized.

The colonoscopy was positive in $96,34 \%$ of cancer cases and falsely negative in $3.65 \%$ cases. Where a sensitivity of $96 \%$ a specificity of $98 \%$ and the predictive positive value and negative 96 percent and 98 percent respectively. Our study is similar to that of Higgin et al. who found a sensitivity $100 \%$, a specificity to $77 \%$ 
[11] [12].

Compared to the treatment (Figure 1), cryotherapy had been carried out at $11.8 \%$ of women with LIEBG and $49.4 \%$ of the LIEHG. The conization was $39 \%$ of the cases of LIEHG and $2.1 \%$ of micro-invasive cancer. The surgery was the treatment in $8.5 \%$ of cancers associated with radiation therapy at $6.4 \%$.

Colposcopy has many advantages in screening for cancer of the cervix. Colposcopy is a reproducible, sensitive and specific screening method.

It is an approach for optimization of the screening and treatment of precancerous lesions, which will allow a wide margin of progress in reducing the incidence and mortality of invasive cervical cancers in countries in the process of development.

\section{Acknowledgements}

The authors thank the head of the francophone center for gynecological cancer screening and treatment and Jhpiego staff for reviewing the findings.

\section{Conflict of Interest}

Contributing authors do not note any conflict of interest in this work. Contributing authors state that they have read and approved the work.

\section{References}

[1] Cartier, R. and Cartier, I. (2005) Colposcopy Convenient. 3rd Edition, 142-143, 146.

[2] Boffetta, P., Gridley, G. and Lindelof, B. (2001) Cancer Risk in a Population-Based Cohort of Patients Hospitalized for Psoriasis in Sweden. Journal of Investigative Dermatology, 117, 1531-1537. https://doi.org/10.1046/j.0022-202x.2001.01520.x

[3] Okonofua, F. (2008) Editorial-HPV Vaccine and Prevention of Odeblad Cancer in Africa. African Journal of Reproductive Health, 11, 7-9. https://doi.org/10.2307/25549711

[4] Reid, R. and Scalzi, P. (1985) Genital Warts and Cervical Cancer. VII. Year Improved Colposcopic Index for Academic Benign Papilloma-Viral Infections from High Grade Cervical Intraepithelial Neoplasia. American Journal of Obstetrics \& Gynecology, 153, 611-618. https://doi.org/10.1016/S0002-9378(85)80244-1

[5] Newfoundland, J., Soft, G., Jones, P.W., et al. (2006) Should Punch Biopsies Be Used when High Grade Disease Is Suspected at Initial Colposcopic Assessment? A Prospective Study. International Journal of Gynecological Cancer, 16, 253-256. https://doi.org/10.1111/j.1525-1438.2006.00344.x

[6] Tebeu, P.M., Sandjong, I., Nkele, N., et al. (2005) Lesions Precancer of the Uterine Cervix in Rural Area: Cross-Sectional Study. Black African Medicine, 52, 1.

[7] Kathys, S., Emmo, O., Patricia, C. and Janet, P. (2007) Supported Comprehensive Reproductive and Sexual Health and Chronic Disease: Fight against Cancer of the Cervix Guide to Essential Practice Geneva: Who.

[8] Ritter, J., Baldauf, J.J. and Dreyfus, M. (Stransbourg) Colposcopy Pubmed.

[9] Dargent, D. (1996) Screening for Cervical Cancer. Point of View of a Gynecologist. Place of the Colposcopy, 83, 742-745.

[10] Rieck, G.C., Bhaumik Beer, J.H.R. and Leeson, S.C. Reopering Cytology at Initial 
Colposcopy Does Not Emprove Detection of High Grade Tail: A Retrospective Cohort Study of 6595 Women.

[11] Higging, R.V., Hall, B., Mc Gee, J.J.A., Lawrence, S., Alvarez, R.D. and Patrdge, E.E. (1994) Apparaisal of the Modelling Used to Evaluate Year Initial Abnormal Pap Smear. Genet Ponocny, 84, 174-178.

[12] Aker, J.C. and Gondry, J. (1998) Precancerous Lesions of the Uterine Cervix. 154-156, 171-172. 